\title{
DYNAMICAL SPACE-TIMES WHICH CONTAIN A CONFORMAL EUCLIDEAN 3-SPACE*
}

BY

\author{
H. P. ROBERTS ON
}

Einstein's theory of gravitation requires that a physical universe be a four-dimensional manifold whose line element is determined by an invariant quadratic differential form whose coefficients $g_{\mu \nu}$ satisfy the ten differential equations

$$
G_{\mu \nu}=\lambda g_{\mu \nu}
$$

in points outside of matter. Here $\lambda$ is a constant and $G_{\mu \nu}$ the contracted Riemann-Christoffel tensor, an expression in the $g_{\mu \nu}$ and their first and second derivatives. $\dagger$

It is the purpose of this paper to restrict the functions $g_{\mu \nu}$ a priori, to solve the equations (0.1) under such restrictions, and to interpret the solutions thus found. In particular, we examine those manifolds which may be described in terms of orthogonal coördinates $x, y, z, t$ such that the 3 -spaces $t=$ constant are conformal euclidean with a ratio of magnification which depends on $t$. The line element of such a manifold may be written

$$
d s^{2}=\frac{1}{\rho^{2}}\left(d x^{2}+d y^{2}+d z^{2}+\sigma^{2} d t^{2}\right)
$$

where $\rho$ and $\sigma$ are functions of all four coördinates and where $\partial \rho(x, y, z, t) / \partial t \neq 0$. If we interpret $t$ as the time coördinate of a physical space-time, then the velocity of light in this space is independent of its direction. $\$$ Physically stated, we propose to find all dynamical space-times which admit of representation by orthogonal coördinates in which the velocity of light is isotropic.

It is not to be expected that all of the solutions found will represent different manifolds, for any transformation under which the line element (0.2) is invariant will carry any one of our solutions into another (or into itself) and in this case the two solutions must be considered as defining

\footnotetext{
* Presented to the Society, San Francisco Section, June 19, 1925; received by the editors in December, 1925.

† The notation here used is that of Eddington's The Mathematical Theory of Relativity, 1923, in which a complete discussion of the theory may be found.

$\ddagger$ Eddington, loc. cit., pp. 40, 93.
} 
the same manifold. Of these transformations the most interesting from the physical standpoint are those which leave the time axis unaltered, thus allowing us to find to what extent the spacial properties are determined for a given observer in a manifold of this type. This requires that the conformal euclidean space $t=$ constant remain conformal euclidean; it has been shown* that the most general transformation satisfying this requirement can be produced by successive applications of linear orthogonal transformations and inversions. We shall find it advantageous to consider as the elemental transformation of this latter group inversion with respect to a point on the circle at infinity, defined by

$$
x=\frac{k x^{\prime}}{y^{\prime}+i z^{\prime}}, \quad y=\frac{r^{\prime 2}-k^{2}}{2\left(y^{\prime}+i z^{\prime}\right)}, \quad z=\frac{r^{\prime 2}+k^{2}}{2 i\left(y^{\prime}+i z^{\prime}\right)}, \quad t=t^{\prime} . \dagger
$$

Under this transformation the line element $(0.2)$ goes into

$$
d s^{2}=\frac{1}{\rho^{\prime 2}}\left(d x^{\prime 2}+d y^{\prime 2}+d z^{\prime 2}+\sigma^{\prime 2} d t^{\prime 2}\right),
$$

where

$$
\rho^{\prime}\left(x^{\prime}, y^{\prime}, z^{\prime}, t^{\prime}\right)=\frac{y^{\prime}+i z^{\prime}}{k} \rho(x, y, z, t), \quad \sigma^{\prime}\left(x^{\prime}, y^{\prime}, z^{\prime}, t^{\prime}\right)=\frac{y^{\prime}+i z^{\prime}}{k} \sigma(x, y, z, t),
$$

in which $x, y, z, t$ are the functions of $x^{\prime}, y^{\prime}, z^{\prime}, t^{\prime}$ defined by (0.3). Hence if we have a solution $\rho(x, y, z, t), \sigma(x, y, z, t)$ the transformation yields another solution $\rho^{\prime}\left(x^{\prime}, y^{\prime}, z^{\prime}, t^{\prime}\right), \sigma^{\prime}\left(x^{\prime}, y^{\prime}, z^{\prime}, t^{\prime}\right)$ which must already be contained among those found by direct methods; we are then justified in limiting ourselves to the discussion of those solutions which are not equivalent under transformations of this type.

1. The equations. The ten equations (0.1) may, in the case of a manifold given by $(0.2)$, be replaced by the set
A
$G_{14}=G_{24}=G_{34}=0$,
B
$G_{23}=G_{31}=G_{12}=0$,
C
$G_{11}=G_{22}=G_{33}$,
D
$G_{11}+G_{22}+G_{33}-\left(1 / \sigma^{2}\right) G_{44}=\left(2 / \rho^{2}\right) \lambda$,
E
$G_{44}=\left(\sigma^{2} / \rho^{2}\right) \lambda$.

* Liouville, Note au sujet de l'article précédent, Journal de Mathématiques, vol. 12 (1847), p. 265.

† Darboux, Systèmes Orthogonaux, 1910, p. 168. 
Expressing the $G_{\mu}$, in terms of $\rho$ and $\sigma$ set A becomes

$$
\rho_{14}-\rho_{4} \frac{\sigma_{1}}{\sigma}=\rho_{24}-\rho_{4} \frac{\sigma_{2}}{\sigma}=\rho_{34}-\rho_{4} \frac{\sigma_{3}}{\sigma}=0,
$$

where the subscripts $1,2,3,4$ indicate differentiation with respect to $x, y, z, t$ respectively. The solution of these equations is

$$
\sigma=\frac{1}{f(t)} \rho_{4},
$$

where $f$ is an arbitrary function of $t$. Using this value of $\sigma$ equations B and $\mathrm{C}$ become

B

$$
\begin{aligned}
& \frac{\rho_{234}}{\rho_{4}}-\frac{2 \rho_{23}}{\rho}=0, \text { etc. } \\
& \frac{\rho_{114}}{\rho_{4}}-\frac{2 \rho_{11}}{\rho}=\frac{\rho_{224}}{\rho_{4}}-\frac{2 \rho_{22}}{\rho}=\text { etc. }
\end{aligned}
$$

whence on integration

$$
\begin{aligned}
\rho_{23} & =\rho^{2} a(x, y, z), \text { etc. }, \\
\rho_{22}-\rho_{33} & =\rho^{2} l(x, y, z), \text { etc. },
\end{aligned}
$$

in which $a, \cdots, l, \cdots$ are arbitrary functions of $x, y$ and $z$. Equations D and $\mathbf{E}$ may also be expressed in terms of $\rho$ and its derivatives; the latter is however not independent ( $D$ is a first integral of it) and may be discarded.

These results may be stated as follows: $A$ manifold whose metrical relations are determined by $(0.2)$ is an Einstein field (i. e., satisfies equations (0.1)) provided

A

$$
\sigma=\frac{1}{f(t)} \rho_{4}, \quad \rho_{4} \neq 0,
$$

$$
\begin{array}{cc}
\text { B } & \rho_{23}=\rho^{2} a(x, y, z), \quad \rho_{31}=\rho^{2} b(x, y, z), \quad \rho_{12}=\rho^{2} c(x, y, z), \\
\text { C } & \rho_{22}-\rho_{33}=\rho^{2} l(x, y, z), \quad \rho_{33}-\rho_{11}=\rho^{2} m(x, y, z), \\
& \quad \rho_{11}-\rho_{22}=\rho^{2} n(x, y, z), \\
\text { D } & -2 \rho\left(\rho_{11}+\rho_{22}+\rho_{33}\right)+3\left(\rho_{1}^{2}+\rho_{2}^{2}+\rho_{3}^{2}\right)=\lambda-3 f^{2}(t),
\end{array}
$$

where $a, b, c, l, m, n$, and $f$ are arbitrary functions of their arguments (except for the identity $l+m+n=0)$. The problem thus reduces to that of the solution of six second-order non-linear partial differential equations in one dependent and three independent variables; we must now investigate under what conditions an integral of these equations may exist. 
2. The conditions of integrability. We next develop certain necessary conditions of integrability for the equations (1.0) above.

First, since

$$
\frac{\partial \rho_{23}}{\partial x}=\frac{\partial \rho_{31}}{\partial y}=\frac{\partial \rho_{12}}{\partial z},
$$

set $B$ requires that

$$
\rho_{1} a+\frac{1}{2} \rho a_{1}=\rho_{2} b+\frac{1}{2} \rho b_{2}=\rho_{3} c+\frac{1}{2} \rho c_{3} .
$$

Next,

$$
\frac{\partial}{\partial x}\left(\rho_{22}-\rho_{33}\right)=\frac{\partial \rho_{12}}{\partial y}-\frac{\partial \rho_{31}}{\partial z},
$$

whence from $B$ and $C$

$$
\rho_{1} l-\rho_{2} c+\rho_{3} b+\frac{1}{2} \rho\left(l_{1}-c_{2}+b_{3}\right)=0 .
$$

To this we must add the two other conditions obtained by the cyclic permutation (123) (abc) (lmn).

The third set of conditions is derived from equation $\mathrm{D}$ in conjunction with $B$ and $C$. With the aid of the second two equations of $C, D$ may be written

$$
-\rho\left(\rho_{22}+\rho_{33}\right)+\frac{1}{3} \rho^{3}(m-n)+\rho_{1}{ }^{2}+\rho_{2}{ }^{2}+\rho_{3}{ }^{2}=\frac{\lambda}{3}-f^{2}(t) .
$$

Differentiating with respect to $x$ and simplifying the resulting equation by means of $B$ we find

$$
b_{3}+c_{2}-\frac{1}{3}\left(m_{1}-n_{1}\right)=0 .
$$

Permutation gives the two other equations of this type.

The conditions (2.1) and (2.2) are five linear homogeneous equations in the four quantities $\rho_{1}, \rho_{2}, \rho_{3}$ and $\rho$, which requires that $a, l$, etc., satisfy the two eliminants of the equations. We may then, in general, solve for the derivatives of $\rho$ and demand that the values thus found are consistent with the original equations; we here develop these additional conditions for the general case $a b c \neq 0$ and consider the alternative cases (which can be handled by simpler methods) as they arise.

In the case here considered $(a b c \neq 0)$ we may eliminate $\rho_{2}$ and $\rho_{8}$ from the first equation of (2.2) by means of (2.1) and obtain as eliminant

$$
\rho_{1} A_{1}^{\prime}+\rho A_{1}=0
$$


where

$$
\begin{aligned}
& A_{1}^{\prime}=b c l-a\left(c^{2}-b^{2}\right), \\
& A_{1}=\frac{1}{2}\left[b c\left(l_{1}-c_{2}+b_{3}\right)-a_{1}\left(c^{2}-b^{2}\right)+b_{2} c^{2}-c_{3} b^{2}\right] .
\end{aligned}
$$

The two remaining equations of (2.2) give similar conditions on $\rho_{2}$ and $\rho_{3}$, in which the coefficients are the cyclic permutations of the above. These equations are equivalent to (2.2) in the case here considered.

In order that (2.1) be consistent with $B$ we must have

$$
\frac{\partial \rho_{2}}{\partial z}=\frac{\partial}{\partial z}\left(\rho_{1} \frac{a}{b}+\rho \frac{a_{1}-b_{2}}{2 b}\right)=\rho^{2} a \text {. }
$$

On eliminating $\rho_{13}$ and $\rho_{3}$ this becomes

$$
\rho_{1} B_{1}^{\prime}+\rho B_{1}=0 \text {, }
$$

where

$$
\begin{aligned}
& B_{1}^{\prime}=a\left(\frac{a_{3}}{a}-\frac{b_{3}}{b}+\frac{a_{1}-b_{2}}{2 c}\right), \\
& B_{1}=\frac{1}{2}\left[\left(a_{1}-b_{2}\right)_{2}+\left(a_{1}-b_{2}\right)\left(\frac{a_{1}-c_{3}}{2 c}-\frac{b_{3}}{b}\right)\right] .
\end{aligned}
$$

Similarly, in order that (2.1) be consistent with C

$$
\frac{\partial \rho_{2}}{\partial y}-\frac{\partial \rho_{3}}{\partial z}=\frac{\partial}{\partial y}\left(\rho_{1} \frac{a}{b}+\rho \frac{a_{1}-b_{2}}{2 b}\right)-\frac{\partial}{\partial z}\left(\rho_{1} \frac{a}{c}+\rho \frac{a_{1}-c_{3}}{2 c}\right)=\rho^{2} l,
$$

and on simplifying as in the previous equation we find

$$
\rho_{1} C_{1}^{\prime}+\rho C_{1}=\rho^{2} \frac{A_{1}^{\prime}}{b c},
$$

where $A_{1}^{\prime}$ is known from (2.21) above and

$$
\begin{aligned}
C_{1}^{\prime}= & \frac{a_{2}}{b}-\frac{a_{3}}{c}+\frac{a}{2 b^{2}}\left(a_{1}-3 b_{2}\right)-\frac{a}{2 c^{2}}\left(a_{1}-3 c_{3}\right), \\
C_{1}= & \frac{1}{2}\left[\frac{1}{b}\left(a_{1}-b_{2}\right)_{2}+\frac{1}{2 b^{2}}\left(a_{1}-b_{2}\right)\left(a_{1}-3 b_{2}\right)\right. \\
& \left.-\frac{1}{c}\left(a_{1}-c_{3}\right)_{2}-\frac{1}{2 c^{2}}\left(a_{1}-c_{3}\right)\left(a_{1}-3 c_{8}\right)\right] .
\end{aligned}
$$

As before, to the equations (2.4) and (2.5) are to be added those obtained by the cyclic permutation (123) (abc) (lmn). 
The 14 equations which have been derived in this section are not independent, but we have written them in this form for the sake of symmetry.

3. Solution of the conditions of integrability. The conditions obtained in the preceding section may be considered as differential equations with dependent variables $\rho, a, l$, etc., which must be solved before we can integrate the original set (1.0). In order to accomplish this it is only necessary to consider two cases: (1), in which the three logarithmic derivatives $\rho_{1} / \rho$, $\rho_{2} / \rho$, and $\rho_{3} / \rho$ of $\rho$ do not depend on $t$, and (2) in which all three of them do. The only alternatives (cases in which one or two only of these three quantities are functions of $t$ ) can be reduced to (2) by rotating the $x, y, z$ coördinate system.

In case (1), $\rho$ must be of the form

$$
\rho=\kappa(t) \tau(x, y, z)
$$

in order that the three logarithmic derivatives be independent of $t$. But the first equation of $(1.0 \mathrm{~B})$ requires that

$$
\tau_{23}=\tau^{2} a \kappa(t)
$$

and since $a$ and $\tau$ cannot depend on $t$ this is only possible if $a=0$. Applying this reasoning to the remaining equations (1.0B) and (1.0C) it is seen that $b, c, l, m$, and $n$ must also vanish, so we have

$$
a=b=c=l=m=n=0
$$

as the solution of the conditions of integrability for this case.

In order to deal with the conditions of integrability for case (2) in the form in which they were developed in $\S 2$ above we first dispose of the possibility $a b c=0$. Then one, and consequently all, of the three quantities $a, b$ and $c$ must vanish; otherwise it would be possible to solve (2.1) for at least one of the logarithmic derivatives of $\rho$ in terms of $x, y$ and $z$ alone, which is contradictory to the assumption that they all depend on $t$. Equations (2.2) then require, for the same reason, that $l, m$ and $n$ also vanish; we thus arrive at the solution (3.1) above.

We may now take as our equations the sets (2.1)-(2.5). Since $a, l$, etc., do not depend on $t$, (2.21) and (2.4) can only be satisfied if all coefficients $A_{1}, A_{1}^{\prime}, B_{1}, B_{1}^{\prime}$ and their cyclic permutations vanish. But then the right hand side of (2.5) is zero, so we must also have $C_{1}=C_{1}^{\prime}=0$, etc. The vanishing of coefficients $A_{\mathfrak{i}}^{\prime}$ enables us to express $l, m$ and $n$ in terms of the variables $a, b$ and $c$ :

$$
l=\frac{a}{b c}\left(c^{2}-b^{2}\right), \quad m=\frac{b}{c a}\left(a^{2}-c^{2}\right), \quad n=\frac{c}{a b}\left(b^{2}-a^{2}\right) .
$$


The conditions of integrability for this case are thus reduced to the 15 equations arising from the vanishing of the coefficients mentioned above, the set (2.3) and the set (2.1). The first 18 of these are, on eliminating $l, m$ and $n$ by (3.2), differential equations with dependent variables $a, b$ and $c$ which are now to be integrated.

12 of these equations (those arising from $A_{i}, B_{i}^{\prime}$, and $C_{i}^{\prime}$, and (2.3)) are of first order, but it is found that of these but 6 are linearly independent. As independent equations we may take two of the set $A_{i}=0$, all of $B_{i}^{\prime}=0$, and one of (2.3):

$$
\begin{gathered}
\frac{b_{1}}{b}+\frac{b_{2}}{2 a}=\frac{c_{1}}{c}+\frac{c_{3}}{2 a}, \\
\frac{c_{2}}{c}+\frac{c_{3}}{2 b}=\frac{a_{2}}{a}+\frac{a_{1}}{2 b}, \\
\frac{a_{3}}{a}+\frac{a_{1}}{2 c}=\frac{b_{3}}{b}+\frac{b_{2}}{2 c}, \\
-a_{1}\left(c^{2}-b^{2}\right)+b_{2} c^{2}-c_{3} b^{2}+b c\left(l_{1}-c_{2}+b_{3}\right)=0, \\
-b_{2}\left(a^{2}-c^{2}\right)+c_{3} a^{2}-a_{1} c^{2}+c a\left(m_{2}-a_{3}+c_{1}\right)=0, \\
c_{2}+b_{3}=\frac{1}{3}\left(m_{1}-n_{1}\right),
\end{gathered}
$$

where $l, m$, and $n$ are given by (3.2). It can be shown that the conditions of complete integrability are not satisfied by this set.*

In order to integrate the second order equations we first note that (3.3) may, in general, be solved for all other derivatives in terms of those with respect to any one, say $z . \dagger$ This being done, it can be shown that

$$
\frac{a_{1}-c_{8}}{2 c}=-\frac{1}{3} \frac{\partial}{\partial z} \log \left(a^{4} b c \Delta^{5 / 2}\right),
$$

where

$$
\Delta=\frac{1}{a^{2}}+\frac{1}{b^{2}}+\frac{1}{c^{2}} .
$$

With the aid of this relation the equation $B_{1}=0((2.4)$ above) becomes

$$
\left(a_{1}-b_{2}\right)_{3}-\frac{1}{3}\left(a_{1}-b_{2}\right) \frac{\partial}{\partial z} \log \left(a^{4} b^{4} c \Delta^{5 / 2}\right)=0,
$$

* Forsyth, Theory of Differential Equations, vol. V, 1902, chap. XI.

$\dagger$ The determinant involved is a multiple of $\Delta$, and the case in which it vanishes is discussed later. 
whence

$$
a_{1}-b_{2}=\left(a^{4} b^{4} c \Delta^{5 / 2}\right)^{1 / 3} \gamma(x, y) \text {. }
$$

Similarly

$$
\begin{aligned}
& b_{2}-c_{3}=\left(a b^{4} c^{4} \Delta^{5 / 2}\right)^{1 / 3} \alpha(y, z), \\
& c_{3}-a_{1}=\left(a^{4} b c^{4} \Delta^{5 / 2}\right)^{1 / 3} \beta(z, x) .
\end{aligned}
$$

On putting the last of these back into $\left(3.3^{\prime}\right)$ we find, introducing the notation $a u=b v=c w=2\left(a b c \Delta^{b / 2}\right)^{-1 / 3}\left(=8 u v w\left(u^{2}+v^{2}+w^{2}\right)^{-5 / 2}\right)$,

$$
\frac{\partial v}{\partial z}=-\beta(z, x)
$$

whence by symmetry considerations

$$
\frac{\partial u}{\partial y}=\gamma(x, y) \text {. }
$$

Four additional equations of this type are obtained by the cyclic permutation (xyz) (uvw) $(\alpha \beta \gamma)$.

The equations $C_{i}=0$ now offer a means of determining the so far arbitrary functions $\alpha, \beta$ and $\gamma ; C_{1}=0$ may be written, after manipulation analogous to that applied above,

$$
\begin{aligned}
& \frac{1}{b}\left(a_{1}-b_{2}\right) \frac{\partial}{\partial y} \log \left[\left(a_{1}-b_{2}\right)\left(a^{4} b^{4} c \Delta^{5 / 2}\right)^{-1 / 8}\right] \\
& -\frac{1}{c}\left(a_{1}-c_{3}\right) \frac{\partial}{\partial z} \log \left[\left(a_{1}-c_{3}\right)\left(a^{4} b c^{4} \Delta^{5 / 2}\right)^{-1 / 3}\right]=0,
\end{aligned}
$$

and on introducing (3.31) this becomes

$$
\frac{\partial \gamma(x, y)}{\partial y}+\frac{\partial \beta(z, x)}{\partial z}=0 .
$$

The remaining equations $C_{2}=C_{3}=0$ yield

$$
\frac{\partial \alpha(y, z)}{\partial z}+\frac{\partial \gamma(x, y)}{\partial x}=0, \quad \frac{\partial \beta(z, x)}{\partial x}+\frac{\partial \alpha(y, z)}{\partial y}=0 .
$$

The solution of these three equations is readily found to be

$$
\alpha=C y-B z+A^{\prime}, \quad \beta=A z-C x+B^{\prime}, \quad \gamma=B x-A y+C^{\prime},
$$

where $A, A^{\prime}$, etc., are constants. 
(3.32) may now be integrated, giving

$$
u=-\frac{1}{2} A r^{2}+x(A x+B y+C z)+C^{\prime} y-B^{\prime} z+X(x),
$$

and two similar equations for $v$ and $w$. Equations (3.31) then become, on using the expressions for $a, b$, and $c$ in terms of $u, v$, and $w$,

$$
u_{1}=v_{2}=w_{3}, \quad \alpha u+\beta v+\gamma w=0 .
$$

The first two of these require that $X^{\prime}(x)=Y^{\prime}(y)=Z^{\prime}(z)$, i. e.

$$
X=-\epsilon x+a^{\prime}, \quad Y=-\epsilon y+b^{\prime}, \quad Z=-\epsilon z+c^{\prime},
$$

where $\epsilon, a^{\prime}, b^{\prime}$ and $c^{\prime}$ are constants; the last relation imposes the conditions

$$
\epsilon A^{\prime}=B c^{\prime}-C b^{\prime}, \quad \epsilon B^{\prime}=C a^{\prime}-A c^{\prime}, \quad \epsilon C^{\prime}=A b^{\prime}-B a^{\prime}
$$

on the constants involved, and if $\epsilon=0$ we must add to these the condition $A A^{\prime}+B B^{\prime}+C C^{\prime}=0$.

The values of $a, b$, and $c$ are now given by

$$
a=\frac{K}{u}, \quad b=\frac{K}{v}, \quad c=\frac{K}{w},
$$

where

$$
\begin{aligned}
K & =2\left(a b c \Delta^{5 / 2}\right)^{-1 / 3}=8 u v w\left(u^{2}+v^{2}+w^{2}\right)^{-5 / 2}, \\
u & =-\frac{1}{2} A r^{2}+x(A x+B y+C z-\epsilon)+C^{\prime} y-B^{\prime} z+a^{\prime}, \text { etc. },
\end{aligned}
$$

and $\epsilon A^{\prime}=B c^{\prime}-C b^{\prime}$, etc.; $A A^{\prime}+B B^{\prime}+C C^{\prime}=0$. These values satisfy the first order equations (3.3) as well as those of second order, and represent the general solution of the conditions of integrability insofar as they do not involve $\rho$ (except for the case $\Delta=0$ ). This solution may be considerably simplified by translation of the $x, y, z$ coördinate system as follows:

(i) The general case in which not all three of the constants $A, B$ and $C$ vanish becomes, on referring it to a coördinate system in which $A^{\prime}, B^{\prime}$ and $C^{\prime}$ vanish,*

$$
u=-\frac{1}{2} A\left(r^{2}-\delta^{2}\right)+x(A x+B y+C z-\epsilon), \text { etc. }
$$

(ii) If $A=B=C=0$ we may write, on translation,

$$
u=C^{\prime} y-B^{\prime} z, \text { etc. }
$$

provided not all $A^{\prime}, B^{\prime}$ and $C^{\prime}$ vanish.

* If, for example, ${ }^{r} A \neq 0$, this may be accomplished by transferring the origin to the point $\left(0,-C^{\prime} / A, B^{\prime} / A\right)$. Further simplification could be accomplished by a rotation, but the symmetry would be destroyed. 
(iii) The only remaining case, that in which all the $A, A^{\prime}$, etc., vanish, is given by

$$
u=-\epsilon x+a^{\prime}, \text { etc. }
$$

In order to determine $\rho$ we must now return to the set (2.1) and substitute in it the known values of $a, b$ and $c$. It then becomes

$$
w \rho_{2}-v \rho_{3}+\alpha \rho=0, v \rho_{3}-w \rho_{1}+\beta \rho=0, \quad v \rho_{1}-u \rho_{2}+\gamma \rho=0,
$$

of which but two equations are independent. On eliminating $\rho$ between two of these we find

$$
\alpha \rho_{1}+\beta \rho_{2}+\gamma \rho_{3}=0,
$$

which may be used in place of one of (3.45) (unless $\alpha, \beta$ and $\gamma$ all vanish). The three cases into which the general solution is divided are the following:

(i) Three independent integrals of the characteristic equations of $\left(3.45^{\prime}\right)$

$$
\frac{d x}{C y-B z}=\frac{d y}{A z-C x}=\frac{d z}{B x-A y}=\frac{d \rho}{0}
$$

are $\rho=$ constant, $A x+B y+C z=$ constant and $x^{2}+y^{2}+z^{2}=r^{2}=$ constant. Hence $\rho=\rho(A x+B y+C z, r, t)$, and (3.45) becomes

$$
(\xi-\epsilon) \rho_{\xi}+\left(\eta-\delta^{2}\right) \rho_{\eta}=\rho,
$$

where $\xi$ and $\eta$ are the two first arguments of $\rho$. The functional form of $\rho$ is consequently given for this case by

$$
\rho=(A x+B y+C z-\epsilon) \tau\left(\frac{r^{2}-\delta^{2}}{A x+B y+C z-\epsilon}, t\right) .
$$

Not all three $A^{2}+B^{2}+C^{2}, \delta^{2}$ and $\epsilon$ may vanish simultaneously, for we would then have $\Delta=0$.

(ii) The values of $a, b$ and $c$ given by (3.42) lead, by the same method, to the solution

$$
\rho=\left(C^{\prime} x-A^{\prime} z\right) \tau\left(\frac{A^{\prime} y-B^{\prime} z}{C^{\prime} x-A^{\prime} z}, t\right) .
$$

(iii) Corresponding to the third case, the equations (3.45) become

$$
\frac{\rho_{1}}{\epsilon x-a^{\prime}}=\frac{\rho_{2}}{\epsilon y-b^{\prime}}=\frac{\rho_{3}}{\epsilon z-c^{\prime}} .
$$

If $\epsilon \neq 0$ these yield

$$
\rho=\rho(r, t) .
$$


In case $\epsilon=0$ the solution is

$$
\rho=\rho(A x+B y+C z, t),
$$

where we have written $A, B$, and $C$ in place of $a^{\prime}, b^{\prime}$ and $c^{\prime}$; these constants are, for the present, subject to the restriction $A^{2}+B^{2}+C^{2} \neq 0$.

The only case remaining to be examined is that in which $\Delta=0$. It can be shown, by a rather tedious analysis which is omitted because of the triviality of the results, that the only solutions arising from this case may be included in (3.51) and (3.54) by removing the restrictions there imposed on the constants $A^{2}+B^{2}+C^{2}, \delta^{2}$ and $\epsilon$. Only the ratios $a: b: c$ are given by (3.33) for these cases.

4. Solution of the equations. We have thus solved the conditions of integrability, and may proceed to the solution of the original equations. Since, however, of the five solutions obtained ((3.1) and (3.51)-(3.54)) the last four are characterized by the fact that in them the functional dependence of $\rho$ is already given, we first examine them in accordance with the remarks made in the introduction to see whether they can lead to distinct manifolds. On subjecting these four solutions to transformations of the form (0.3) there result solutions (0.31) which are already among the four; in particular, it can be shown that (3.52)-(3.54) can be obtained from (3.51) (or special cases thereof), and that (3.53) and (3.54) are together fully equivalent to it. It is therefore only necessary to consider solutions of (1.0) arising from (3.1) and (3.51) or (and this is the course we shall follow) those arising from (3.1), (3.53) and (3.54).

In the first case to be considered the six quantities $a \cdots l \cdots$ vanish, so the first five equations of (1.0) are

$$
\rho_{23}=\rho_{31}=\rho_{12}=0, \quad \rho_{11}=\rho_{22}=\rho_{33} .
$$

The solution of these is

$$
\rho=d(t)\left(x^{2}+y^{2}+z^{2}\right)+d_{1}(t) x+d_{2}(t) y+d_{3}(t) z+d_{4}(t),
$$

where the $d$ 's are arbitrary functions. (1.0D) requires that

$$
f^{2}(t)=\frac{\lambda}{3}-d_{1}^{2}-d_{2}^{2}-d_{3}^{2}+4 d d_{4},
$$

and on introducing these values of $\rho$ and $f(t)$ into (1.0A) we obtain $\sigma$. Substituting $\rho$ and $\sigma$ into the components of the Riemann-Christoffel tensor $B_{\alpha \beta \gamma \delta}{ }^{*}$ we find that the manifold defined by this case is a hypersphere of

* Eddington, loc. cit., p. 72. 
Riemann curvature $-\lambda / 3$. This solution is equivalent to one obtained by E. Kasner.*

It is readily shown that the only manifolds of type (0.2) in which the coefficient of $d t^{2}$ is a function of $t$ alone are special cases of (4.1); we may deduce from this the impossibility of stationary observers (as their existence implies that the world is not "empty") employing orthogonal coordinates, of which their proper time is one, in which the velocity of light is isotropic, unless their fundamental intervals are functions of their position.

For the second case, (3.53), in which $\rho$ is a function of $r$ and $t$, the equations (1.0) are

$$
\begin{gathered}
\frac{\partial^{2} \rho}{\partial r^{2}}-\frac{1}{r} \frac{\partial \rho}{\partial r}=\left(\frac{2}{\epsilon}\right)^{8} \frac{\rho^{2}}{r^{3}}, \\
-\frac{2}{3} \rho \frac{\partial^{2} \rho}{\partial r^{2}}-\frac{4}{3} \frac{\rho}{r} \frac{\partial \rho}{\partial r}+\left(\frac{\partial \rho}{\partial r}\right)^{2}=\frac{\lambda}{3}-f^{2}(t) .
\end{gathered}
$$

On eliminating the second derivative of $\rho$ these lead to the equivalent first order equation

$$
\left(\frac{\partial \rho}{\partial r}\right)^{2}-2 \frac{\rho}{r} \frac{\partial \rho}{\partial r}-4 C \frac{\rho^{8}}{r^{3}}=\frac{\lambda}{3}-f^{2}(t)
$$

where $C=(4 / 3)\left(1 / \epsilon^{3}\right)$. The solution of this is

$$
\rho=\frac{r}{C}\left\{\varphi(\log r g(t))-\frac{1}{12}\right\}
$$

in which $\varphi$ is a Weierstrass $\wp$-function whose invariants are

$$
g_{2}=\frac{1}{12}, \quad g_{3}=-\frac{1}{216}-\left(\frac{\lambda}{3}-f^{2}(t)\right) C^{2} .
$$

In this form the line element contains elliptic functions, and it is difficult to reconcile their doubly-periodic property with any attempted physical interpretation; $\nmid$ as will be shown below, however, this behavior

* E. Kasner, American Journal of Mathematics, vol. 43 (1921), pp. 20-28; Mathematische Annalen, vol. 85 (1922), pp. 234-236.

$\dagger$ The difficulty of this reconciliation can be seen from the fact that although $\varnothing$ need not be periodic in $\log r$, it must at least be possible to find at any time $t$ values for which $|\wp|$ assumes any assigned value. Two integrals of the equations of motion of a particle placed in this field are

$$
\theta=\frac{\pi}{2}, \quad\left(\frac{r}{\rho}\right)^{2} \frac{d \varphi}{d s}=\left(\frac{C}{\rho}\right)^{2} \frac{d \varphi}{d s}=\text { const. }
$$

and these, because of the above mentioned property of $\varphi$, would require a rather erratic behavior. (Cf. Eddington, loc. cit., pp. 60, 85.) 
is not inherent in the manifold, but is a property of the coördinate system we have chosen. In order to avoid this objection we require that $p$ degenerate to a singly periodic function in such a way that it is not periodic along the real axis; this is only possible if we take the root $f^{2}(t)=\lambda / 3$ of the condition $g_{2}^{2}-27 g_{2}^{2}=0 . \rho$ is then, on integration,

$$
p=\frac{1}{1} \tanh ^{2} \log (r g(t))^{1 / 2}-\frac{1}{6},
$$

and on simplifying and substituting in (4.2),

$$
\rho=-\frac{1}{C g(t)}\left(1+\frac{1}{r g(t)}\right)^{-2}
$$

$t$ only appears in $\rho$ in the parameter $g(t)$, so the coefficient of $d t^{2}$ in the line element contains $\left[g^{\prime}(t)\right]^{2}$ as a factor; hence no loss of generality is incurred by choosing $g$ in any way we please. In particular, we write $C=i m / 2$ and choose $g(t)=(2 / m) e^{x t}$ where $\kappa^{2}=\lambda / 3$. Then

$$
\begin{gathered}
d s^{2}=-e^{-2 x t}\left(e^{x t}+\frac{m}{2 r}\right)^{4}\left(d r^{2}+r^{2} d \theta^{2}+r^{2} \sin ^{2} \theta d \phi^{2}\right) \\
+\frac{\left(e^{x t}-\frac{m}{2 r}\right)^{2}}{\left(e^{x t}+\frac{m}{2 r}\right)^{2}} d t^{2} .
\end{gathered}
$$

The manifold defined by (4.21) reduces to a special case of (4.1), and therefore represents a hypersphere, if $m=0$. If $k=0$ the coefficients, although no longer containing $t$, still satisfy the equations $(0.1)$; the line element is, in fact, the isotropic form of the Schwarzschild element for that case. ${ }^{*}$ On the other hand, it is well known that the general Schwarzschild solution

$$
\begin{gathered}
d s^{2}=-\left(\frac{d R^{2}}{\gamma}+R^{2} d \Theta^{2}+R^{2} \sin ^{2} \Theta d \Phi^{2}\right)+\gamma d T^{2}, \\
\gamma=1-\frac{2 m}{R}-\kappa^{2} R^{2}
\end{gathered}
$$

represents the same fields in these two limiting cases; it therefore seems reasonable to ask if (4.21), or the apparently more general (4.2) from which it was derived, is not equivalent to the Schwarzschild element (4.2'). That

\footnotetext{
- For this and the following, see Eddington, loc. cit., pp. 93, 100.
} 
this is, in fact, the case can be shown by subjecting $\left(4.2^{\prime}\right)$ to the transformation

$$
R=R(r, t), \quad \Theta=\theta, \quad \Phi=\phi, \quad T=T(r, t),
$$

and requiring that the resulting expression be of the form (0.2). (4.2') then becomes (the subscripts $r$ and $t$ denoting differentiation)

$$
d s^{2}=-\frac{R^{2}}{r^{2}}\left(d r^{2}+r^{2} d \theta^{2}+r^{2} \sin ^{2} \theta d \phi^{2}\right)+\left(\gamma T_{t}^{2}-\frac{1}{\gamma} R_{t}^{2}\right) d t^{2},
$$

provided $R$ and $T$ satisfy

$$
\gamma T_{r} T_{t}=\frac{1}{\gamma} R_{r} R_{t}, \quad \frac{R_{r}^{2}}{\gamma}-\gamma T_{r}^{2}=\frac{R^{2}}{r^{2}} .
$$

On solving these two equations for $T_{r}$ and $T_{t}$ and applying the condition of integrability

$$
\frac{\partial T_{r}}{\partial t}=\frac{\partial T_{t}}{\partial r}
$$

we obtain

$$
r^{2} R R_{r r}-2 r^{2} R_{r}^{2}+r R R_{r}-\frac{1}{2} \gamma^{\prime} R^{3}+\gamma R^{2}=0, \quad \gamma^{\prime}=\frac{d \gamma}{d R},
$$

whence, on multiplying by $2 R_{r} / R^{5}$ and integrating with respect to $r$,

$$
\frac{r^{2} R_{r}^{2}}{R^{4}}-\frac{\gamma}{R^{2}}=f^{2}(t)
$$

a function of $t$ alone. Changing from dependent variable $R$ to $\rho=i r / R$ this equation becomes

$$
\left(\frac{\partial \rho}{\partial r}\right)^{2}-2 \frac{\rho}{r} \frac{\partial \rho}{\partial r}-2 m i\left(\frac{\rho}{r}\right)^{3}=\frac{\lambda}{3}-f^{2}(t),
$$

and the coefficient of $d t^{2}$

$$
\left(\frac{\partial \rho}{\partial t}\right)^{2} \frac{1}{\rho^{2} f^{2}(t)}
$$

But the line element defined in this case is seen to be identical with (4.2) on setting $m=-2 i C$. Hence our "dynamical" spherically symmetric solution is equivalent to the Schwarzschild statical solution! 
The line element (4.21) is equivalent to the one defined by (4.2), from which it was obtained by setting $f^{2}(t)=\lambda / 3$, since the latter is equivalent to $\left(4.2^{\prime}\right)$ for all values of $f(t)$. It can be obtained directly from the Schwarzschild form by the transformation

$$
\begin{aligned}
& R=r e^{-k t}\left(e^{k t}+\frac{m}{2 r}\right)^{2}, \quad \Theta=\theta, \quad \Phi=\phi, \\
& T=t+8 m^{2} k F\left(\frac{e^{k t}-m /(2 r)}{e^{k t}+m /(2 r)}\right),
\end{aligned}
$$

where

$$
F(\xi)=\int \frac{d \xi}{\left(1-\xi^{2}\right)\left[\xi^{2}\left(1-\xi^{2}\right)^{2}-4 m^{2} \kappa^{2}\right]} .
$$

The evaluation of $F(\xi)$ involves the solution of the cubic equation $\xi^{2}-\xi \pm 2 m \kappa=0$, and is not necessary for our purpose.

The last case, $\rho=\rho(A x+B y+C z, t)$, leads to two solutions, according as $A^{2}+B^{2}+C^{2}$ vanishes or not. In the latter case we may, on rotation of the coördinate system, bring $\rho$ into the form $\rho=\rho(x, t)$. Equation (1.0) then becomes

$$
\left(\frac{\partial \rho}{\partial x}\right)^{2}=4 C \rho^{3}+\frac{\lambda}{3}-f^{2}(t)
$$

of which the solution is

$$
\begin{aligned}
& \rho=\varphi\left(C^{1 / 2} x+g(t)\right), \\
& g_{2}=0, \quad g_{3}=-\frac{1}{C}\left(\frac{\lambda}{3}-f^{2}(t)\right) .
\end{aligned}
$$

Using the methods employed in the preceding case, it can be shown that this solution is equivalent to

$$
d s^{2}=\frac{4 d X^{2}}{K^{2}\left(1+X^{2}\right)^{2}}+\left(\frac{2}{1+x^{2}}\right)^{2 / 3}\left(d Y^{2}+d Z^{2}+X^{2} d T^{2}\right), \quad K^{2}=-9 \kappa^{2},
$$

which is a special case of a solution obtained by $E$. Kasner. ${ }^{*}$ As before, we may choose $f^{2}(t)=\lambda / 3$, and obtain the equivalent solution

$$
d s^{2}=(x-\kappa t)^{4}\left(d x^{2}+d y^{2}+d z^{2}\right)+4(x-\kappa t)^{-2} d t^{2} .
$$

The case $k=0$ of this solution has been given explicitly by Kasner.

* E. Kasner, these Transactions, vol. 27 (1925), p. 155. 
If $A^{2}+B^{2}+C^{2}=0$, we may take $\rho$ in the form $\rho=\rho(x+i y, t)$. Then (1.0) becomes

$$
\frac{\partial^{2} \rho}{\partial \xi^{2}}=\phi(\xi) \rho^{2}, \quad \xi=x+i y,
$$

where $\phi$ is arbitrary and $f^{2}(t)=\lambda / 3$. It is not possible to give this solution a physical interpretation if we consider $x, y$ and $z$ as the space coördinates, for the coefficients of the line element would then be functions of a complex variable. In order to avoid this we could interchange the rôle of $x$ or $y$ with $t$, but the velocity of light would no longer be isotropic.

In conclusion, we have found that there exist four distinct Einstein fields whose line elements are of the form (0.2), given by (4.1), (4.21), (4.31) and (4.4). These represent (1) a hypersphere, (2) a dynamical form of the statical Schwarzschild solution, (3) a dynamical form of a solution given by Kasner, a "one-dimensional state of motion," and (4) a rather general class given by the solution of any equation of the type (4.4), which involves one essential arbitrary function of $t$. The question of whether we may consider the $r$ and $t$ of (2) as physically observed quantities has not been here considered, nor have we attempted to determine whether all statical solutions of the form (0.2) may on transformation be thrown into the dynamical form, as was done in this case.

\section{Calmorna Institute or Technology,} Pasadena, Calu. 